\title{
Adaptive Disturbance Torque Estimation for Orbiting Spacecraft Using Recursive Least-Squares Methods
}

\author{
Sean Shan-Min Swei, ${ }^{*}$ Nhan T. Nguyen ${ }^{\dagger}$ \\ NASA Ames Research Center, Moffett Field, CA 94035-1000
}

\begin{abstract}
This paper develops a novel disturbance torque estimator for an orbiting spacecraft by utilizing the adaptive least-squares parameter estimation technique. The disturbance estimation is first formulated as an adaptive least-squares minimization problem using a set of polynomial functions, and then integrated with the feedback momentum estimator. The covariance update law with a variable forgetting factor is used and it is shown that the convergent rate for estimation errors can be made at the same level as the forgetting factor. The proposed approach is particularly suited for orbiting small or micro satellite applications, where the momentum management capacity is often limited. The on-board estimated disturbance torque input can then be used as a part of control resource for spacecraft momentum management. The simulation results demonstrate the efficacy of the proposed concept.
\end{abstract}

*Research Scientist, Intelligent Systems Division, Senior Member.

${ }^{\dagger}$ Research Scientist, Intelligent Systems Division, Associate Fellow. 


\section{Nomenclature}

\begin{tabular}{|c|c|c|}
\hline${ }^{I} H^{s p}$ & $=$ & $\begin{array}{l}\text { spacecraft rigid body angular momentum (including the stationary reaction wheels) } \\
\text { relative to the inertial frame, Nms }\end{array}$ \\
\hline$J_{s}$ & $=$ & spacecraft mass moment of inertia, including the stationary reaction wheels, $\mathrm{kg}-\mathrm{m}^{2}$ \\
\hline${ }^{I} \omega^{b}$ & $=$ & spacecraft angular velocity relative to the inertial frame, $\mathrm{rad} / \mathrm{sec}$ \\
\hline${ }^{b} \omega^{r w}$ & $=$ & reaction wheel angular velocity relative to the body-fixed frame, $\mathrm{rad} / \mathrm{sec}$ \\
\hline$T$ & $=$ & $3 \times 4$ conversion matrix from reaction wheel axial unit vectors to the body-fixed fra \\
\hline$I_{i}$ & $=$ & $i$ th reaction wheel axial moment of inertia, $\mathrm{kg}-\mathrm{m}^{2}$ \\
\hline$\Omega_{i}$ & $=$ & $i$ th reaction wheel spin rate relative to the body-fixed frame, $\mathrm{rad} / \mathrm{sec}$ \\
\hline$\beta$ & $=$ & reaction wheel base angle, rad \\
\hline${ }^{I} H^{r w}$ & $=$ & total spinning reaction wheel angular momentum relative to the inertial frame, Nms \\
\hline${ }^{I} H^{s s}$ & $=$ & total angular momentum for the spacecraft system relative to the inertial frame, $\mathrm{Nm}$ \\
\hline$f$ & $=$ & environmental disturbance torque, $\mathrm{Nm}$ \\
\hline$\hat{f}$ & $=$ & estimated environmental disturbance torque, $\mathrm{Nm}$ \\
\hline$q_{i}, i=1, \cdots, 4$ & $=$ & quaternion elements \\
\hline$h_{r w}$ & $=$ & reaction wheel angular momentum along its spin axis, Nms \\
\hline$h$ & $=$ & total reaction wheel angular momentum relative to the body-fixed frame, Nms \\
\hline$u$ & $=$ & control input \\
\hline$H$ & $=$ & spacecraft angular momentum, Nms \\
\hline$\hat{H}$ & $=$ & estimated spacecraft angular momentum, Nms \\
\hline$\phi_{i}$ & $=$ & basis functions \\
\hline$\Theta$ & $=$ & unknown constant matrix \\
\hline$\hat{\Theta}$ & $=$ & estimation of $\Theta$ \\
\hline$\alpha$ & $=$ & forgetting factor or rate of convergence \\
\hline$\left(X_{B}, Y_{B}, Z_{B}\right)$ & $=$ & spacecraft body-fixed frame \\
\hline$(X, Y, Z)$ & $=$ & inertial reference frame \\
\hline
\end{tabular}

\section{Introduction}

Estimation of disturbance input is a subject of practical significance in space applications, especially for small or micro satellites conducting science missions near earth or lunar orbits subjected to unknown disturbance inputs due to, for example, aerodynamic drag, magnetic field or gravity gradient. For on-orbit guidance, navigation and control (GN\&C) mission planning, these external torques are taken into consideration when determining the 
momentum management operations during space science missions, as these would impact the capacity and size of the momentum management actuators, such as reaction wheels and/or reaction control system (RCS) thrusters. For large missions conducted by sizable spacecraft, sufficient margins are often built into the design and selection of actuators. However, for a small or micro satellite application, such as the Lunar Atmosphere and Dust Environment Explorer $(\mathrm{LADEE})^{1}$ or CubeSats, ${ }^{2}$ because of its low budget and high risk nature of missions, the amount of science and the level of precision it can achieve are limited by the available actuation control authority and the disturbance environment it is exposed to.

The problem of designing an observer for linear systems with unknown inputs has been studied quite extensively in the past, ${ }^{3-7}$ where the so-called unknown input observer (UIO) was developed to estimate the system states through the decomposition of the state vector according to the presence of disturbance input. On the other hand, the subject of disturbance input estimation is often motivated by practical needs; for example, monitoring and controlling the cutting force in machine tooling ${ }^{8,9}$ and for system fault detection and isolation. ${ }^{10,11}$ The common goal in these considerations is to improve the performance of the overall system behavior by making use of the estimated disturbance input. Of course, when the disturbance input can be modeled through an input-output relationship, it can be readily incorporated into the plant dynamics and considered as part of the overall system description. However, for an unknown and not measurable disturbance input, various approaches have been proposed. For a more general multi-input multi-output system consideration, Corless and $\mathrm{Tu},{ }^{12}$ and $\mathrm{Ha}$ and $\mathrm{Trinh}^{13}$ developed a disturbance input estimator by utilizing the linear matrix inequality (LMI) technique for a class of nonlinear/uncertain systems satisfying a Lipschitz condition. Their design does not involve differentiating the measured outputs, as opposed to Park and Stein ${ }^{8}$ where a disturbance estimator was designed by differentiating the output measurement. Xiong and Saif ${ }^{14}$ extended the disturbance estimation design by proposing a reduced order input estimator and an adaptive observer design for linear time-invariant systems. Liu and Peng ${ }^{15}$ designed a disturbance observer by utilizing a dynamic inversion algorithm and by enforcing prescribed algebraic conditions on input and output matrices through the selection of some parameter matrices. In this paper, we propose to design an integrated adaptive disturbance torque estimator and momentum observer for an orbiting spacecraft. The goal is to utilize the disturbance torque through adaptive identification as a source of spacecraft momentum management authority during orbital science missions. In particular, it can be used to desaturate the reaction wheel momentum, unlike conventional momentum dumping maneuvers that rely on the execution of RCS thrusters. This approach is especially appealing for small or micro satellite applications, where the impact of disturbance torque is more pronounced because of its limited control authority.

In this paper, a polynomial approximation for an unknown disturbance input is formu- 
lated using least-squares methods. ${ }^{16,17}$ Specifically, fixed order Chebyshev polynomials are used as basis functions, and an adaptive momentum estimator is developed and integrated with spacecraft rigid-body equations of motion to form an augmented disturbance torque estimation system. The covariance update law that has a variable forgetting factor is implemented $^{17}$ and, by using a Lyapunov-type approach, it can be shown that the rate of estimation error convergence for both disturbance and momentum estimation can be made at the same level as the forgetting factor.

The rest of the paper is organized as follows. In Section II, we consider the dynamics of an orbiting spacecraft that has four reaction wheels. A momentum reduction scheme is proposed that suggests a range of spacecraft attitude maneuver in order to take advantage of the estimated disturbance torque. An adaptive least-squares approach is introduced in Section III, where we utilize the notion of quadratic stability to demonstrate that the rate of convergence for disturbance estimation error is guaranteed to be the same as the forgetting factor. It is also shown that the achievable convergent rate or forgetting factor is determined only when a positive definite solution exists for an associated time-varying Riccati differential equation. In Section IV a momentum estimator for an orbiting spacecraft is developed for estimating the spacecraft angular momentum. Using Lyapunov stability theory it is shown that the disturbance torque estimation error and the momentum estimation error converge to zero simultaneously at a prescribed rate of convergence given by the forgetting factor. Section V contains a numerical simulation. There we consider a lunar orbiting small spacecraft, where the dominant disturbance torque is due to the lunar gravity gradient. The proposed adaptive disturbance estimator is demonstrated to be effective in predicting the disturbance torques. In addition, the estimated disturbance torque is then used to desaturate the reaction wheels. Finally, Section VI contains a summary conclusion.

\section{Spacecraft System Dynamics}

In this paper, we consider an orbiting small or micro spacecraft whose attitude maneuvers are achieved by the onboard reaction wheels. For the purpose of this study, we assume there are four reaction wheels and they are arranged in such a way to produce sufficient control torque authority and also provide redundancy. This reaction wheel arrangement has commonly been adopted by many space missions, such as LADEE, ${ }^{1}$ Kepler, ${ }^{18}$ Lunar Reconnaissance Orbiter (LRO), ${ }^{19}$ and Solar Dynamic Observatory (SDO) ${ }^{20}$ to name a few. For illustration, Fig. 1 shows an example of four reaction wheels placed in a four-sided pyramid configuration with base angle $\beta .^{21}$ The angular momentum of the spacecraft in the inertial frame is given by 


$$
{ }^{I} H^{s p}(t)=J_{s}^{I} \omega^{b}(t)
$$

where $J_{s}$ is the mass moment of inertia of the spacecraft, including the stationary reaction wheels, and ${ }^{I} \omega^{b}=\left[\omega_{x} \omega_{y} \omega_{z}\right]^{T}$ is the angular velocity of the spacecraft relative to the inertial frame. Let $T$ be the conversion matrix that transforms the reaction wheel spin axis to the spacecraft body-fixed frame, and is given by

$$
T=\left[\begin{array}{cccc}
\cos \beta & 0 & -\cos \beta & 0 \\
0 & \cos \beta & 0 & -\cos \beta \\
\sin \beta & \sin \beta & \sin \beta & \sin \beta
\end{array}\right]
$$

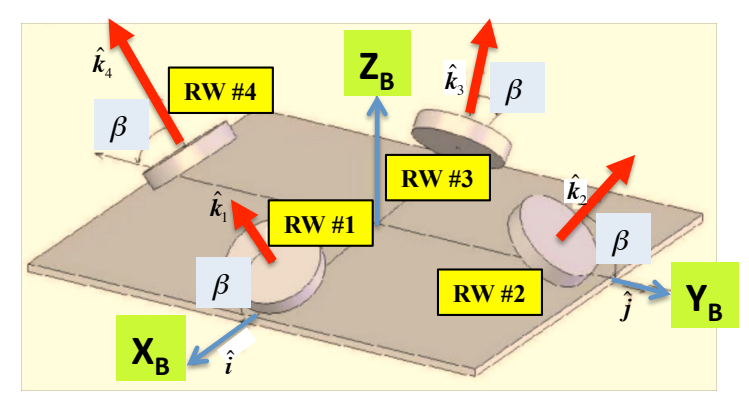

Figure 1. A pyramid configuration of four reaction wheels.

Then, the total reaction wheel angular momentum relative to the inertial frame, denoted by ${ }^{I} H^{r w}$, can be described in the spacecraft body-fixed frame as

$$
{ }^{I} H^{r w}(t)=T I_{r w} T^{T I} \omega^{b}(t)+T I_{r w}^{b} \omega^{r w}(t)
$$

where $I_{r w}=\operatorname{diag}\left\{I_{1}, I_{2}, I_{3}, I_{4}\right\} ; I_{i}$ denotes the $i$ th wheel axial moment of inertia, and ${ }^{b} \omega^{r w}=\left[\Omega_{1}, \Omega_{2}, \Omega_{3}, \Omega_{4}\right]^{T} ; \Omega_{i}$ denotes the $i$ th wheel spin rate relative to the body-fixed frame.

Therefore, the total angular momentum for the spacecraft system is the summation of the spacecraft angular momentum given in Eq. (1) and reaction wheel angular momentum 
given in Eq. (3), and is described by

$$
\begin{aligned}
{ }^{I} H^{s s}(t) & ={ }^{I} H^{s p}(t)+{ }^{I} H^{r w}(t) \\
& =J_{s}{ }^{I} \omega^{b}(t)+T I_{r w} T^{T I} \omega^{b}(t)+T I_{r w}{ }^{b} \omega^{r w}(t) \\
& =J^{I} \omega^{b}(t)+T I_{r w}{ }^{b} \omega^{r w}(t),
\end{aligned}
$$

where $J=J_{s}+T I_{r w} T^{T}$. Note that in the case of large spacecraft the reaction wheel inertia can be negligible compared to the spacecraft inertia, hence $J \approx J_{s}$. However, this may not be the case for micro spacecraft, where size/mass of the reaction wheel is comparable to the size/mass of the spacecraft. The derivation for the rotational equations of motion follows a standard approach, ${ }^{21-23}$ for completeness we present below a derivation.

Let $f(t)$ denote the total external disturbance torque, and by taking the time derivative of Eq. (4) with respect to the inertial frame and setting it equal to the external disturbance torque, we obtain

$$
\begin{aligned}
f(t) & =\frac{{ }^{I} d^{I} H^{s s}(t)}{d t} \\
& =\frac{{ }^{b} d^{I} H^{s s}(t)}{d t}+{ }^{I} \omega^{b}(t) \times{ }^{I} H^{s s}(t) .
\end{aligned}
$$

In attaining above we have applied the fundamental kinematics equation to obtain the second equality, where $\frac{{ }^{b} d X}{d t}$ denotes the time derivative of $X$ with respect to the spacecraft body-fixed frame. Substituting Eq. (4) into above yields,

$$
\begin{aligned}
f(t) & =\left[J^{I} \dot{\omega}^{b}(t)+T I_{r w}{ }^{b} \dot{\omega}^{r w}(t)\right]+{ }^{I} \omega^{b}(t) \times\left[J^{I} \omega^{b}(t)+T I_{r w}{ }^{b} \omega^{r w}(t)\right] \\
& =\underbrace{\left[J^{I} \dot{\omega}^{b}(t)+{ }^{I} \omega^{b}(t) \times J^{I} \omega^{b}(t)\right]}_{\text {Spacecraft }}+\underbrace{\left[T I_{r w}{ }^{b} \dot{\omega}^{r w}(t)+{ }^{I} \omega^{b}(t) \times T I_{r w}{ }^{b} \omega^{r w}(t)\right]}_{\text {Reaction Wheels }} .
\end{aligned}
$$

Let $u(t)=\left[\begin{array}{lll}\tau_{x} & \tau_{y} & \tau_{z}\end{array}\right]^{T}$ be the internal control torque generated by the reaction wheels, then the equal and opposite torque will be applied to the spacecraft. Therefore, Eq. (6) can be rewritten as follows,

$$
J^{I} \dot{\omega}^{b}(t)=J^{I} \omega^{b}(t) \times{ }^{I} \omega^{b}(t)+u(t)+f(t),
$$

where the control torque $u(t)$ is defined by

$$
\begin{aligned}
u(t) & =-T I_{r w}{ }^{b} \dot{\omega}^{r w}(t)-{ }^{I} \omega^{b}(t) \times T I_{r w}{ }^{b} \omega^{r w}(t) \\
& =-T \dot{h}_{r w}(t)-{ }^{I} \omega^{b}(t) \times T h_{r w}(t),
\end{aligned}
$$

where $\dot{h}_{r w}(t)$ and $h_{r w}(t)$ denote the reaction wheel torque and angular momentum along its spin axis. Eq. (7) represents the spacecraft dynamics subject to the control torque and the external disturbance torque. 


\section{II.A. Attitude kinematic equations}

The spacecraft attitude kinematics can be expressed in terms of quaternions, and hence the quaternion kinematic differential equations can be described by ${ }^{22}$

$$
\dot{\hat{q}}=\frac{1}{2} \Lambda \hat{q}
$$

where $\hat{q}=\left[\begin{array}{llll}q_{1} & q_{2} & q_{3} & q_{4}\end{array}\right]^{T}$ denotes the quaternion vector and $\Lambda$ is defined as

$$
\Lambda=\left[\begin{array}{cccc}
0 & \omega_{z} & -\omega_{y} & \omega_{x} \\
-\omega_{z} & 0 & \omega_{x} & \omega_{y} \\
\omega_{y} & -\omega_{x} & 0 & \omega_{z} \\
-\omega_{x} & -\omega_{y} & -\omega_{z} & 0
\end{array}\right]
$$

Let $H=J^{I} \omega^{b}$ denote the spacecraft system momentum, then Eq. (7) along with Eq. (9) forms a complete equations of motion for spacecraft attitude control during orbital maneuvers,

$$
\Sigma:\left\{\begin{array}{l}
\dot{H}(t)=H(t) \times \omega(t)+u(t)+f(t) \\
\dot{\hat{q}}(t)=\frac{1}{2} \Lambda(t) \hat{q}(t)
\end{array}\right.
$$

To simplify the presentation, we have dropped the superscripts. The goal of this paper is to design an estimator for disturbance torque $f(t)$, therefore it can be used to assist spacecraft momentum management and attitude control. We first propose to utilize an adaptive least-squares minimization approach to approximate the disturbance torque using a set of regression functions, and then integrate it with the feedback estimator for momentum estimation. The attitude (quaternions) measurement data will be recorded and used to characterize the disturbance torque, and can be used to characterize the relation between spacecraft attitude and disturbance torque.

\section{II.B. A momentum reduction scheme}

In most spacecraft attitude control problems, the external/environmental disturbances were

omitted from the problem formulation ${ }^{22,23}$. This is justified since the reaction wheels are often used for attitude controls, and the excessive angular momentum caused by the external disturbances can be directly transferred and stored in the reaction wheels. Therefore, as long as there is sufficient reaction wheel momentum capacity to provide the necessary control authority, the disturbance torque can be omitted when designing the attitude controllers. For many spacecraft missions, activation of RCS thrusters is used to perform a planned momentum reduction maneuver for removal of excessive reaction wheel angular momentum. 
In this paper, we utilize the estimated disturbance torque to unload the reaction wheel angular momentum. To this end, we assume that both spacecraft attitude and disturbance torque are available, hence, depending on the direction of the reaction wheel momentum build-up, we may re-orient the spacecraft attitude so that the estimated disturbance torque $f(t)$ is aligned opposite to the direction of the momentum build-up. The following derivation illustrates the proposed approach.

Recall Eqs. (7) and (8). If we set $u(t)+f(t)=0$, from Eq. (8), we obtain

$$
f(t)=\dot{h}(t)+\omega(t) \times h(t)
$$

where $h(t)=T h_{r w}(t)$ denotes the total reaction wheel angular momentum relative to the body-fixed frame. To determine the direction of $f(t)$ for momentum management, let $V(t)=$ $h^{T}(t) h(t)$ be a candidate Lyapunov function for (11). Then, by taking the time derivative of $V(t)$ along any solution of (11), yields

$$
\dot{V}(t)=2 h^{T}(t) \dot{h}(t)=2 h^{T}(t)[f(t)-\omega(t) \times h(t)] .
$$

Since $h(t)$ is orthogonal to $\omega(t) \times h(t),(12)$ can be simplified to

$$
\dot{V}(t)=2 h^{T}(t) f(t) \text {. }
$$

It is obvious from (13) that, in order to ensure $\dot{V}(t)<0$ or equivalently the monotonic decreasing of reaction wheels momentum, the angle $\theta$ between the two vectors $h(t)$ and $f(t)$ must satisfy: $\frac{\pi}{2}<\theta<\frac{3 \pi}{2}$. In addition, the maximum momentum reduction can be achieved when the spacecraft attitude is re-oriented such that $f(t)$ is aligned with $-h(t)$, that is $\theta=\pi$.

In what follows, we present the disturbance torque estimation by utilizing the adaptive least-squares approach, integrated with an angular momentum estimator.

\section{Adaptive Least-Squares Method}

Before proceeding further, we consider the following nonlinear system,

$$
\dot{x}(t)=F(x(t))
$$

where $x \in R^{n}$ and the function $F(\cdot)$ is assumed continuous. The following definition is common in the literature on robust stabilization via Lyapunov functions, see for instance Corless $^{24}$ and Swei and Corless. ${ }^{25}$ 
Definition 1: Let $\alpha>0$ be given. The system described in Eq. (14) is quadratically stable at a rate of convergence $\alpha$, if there exists a positive-definite symmetric matrix $P \in$ $R^{n \times n}$ such that

$$
x^{T} P F(x) \leq-\alpha x^{T} P x,
$$

for all $x \in R^{n}$.

It should be noted that if there is a $P>0$ satisfying Eq. (15), then $V(x)=x^{T} P x$ is a Lyapunov function for Eq. (14), since in this case,

$$
\begin{aligned}
\dot{V}(x) & =2 x^{T} P F(x) \\
& \leq-2 \alpha x^{T} P x \\
& =-2 \alpha V(x)<0,
\end{aligned}
$$

which, in fact, indicates that Eq. (14) is globally uniformly exponentially stable with a rate of convergence $\alpha .^{24,25}$

Recall Eq. (10). Let the disturbance input $f(t)$ be given by

$$
f(t)=\Theta^{T} \Phi(t)
$$

where $\Theta$ is an unknown constant matrix and $\Phi(t)$ a known vector of basis functions, such as Chebyshev polynomials. Let $\Phi(t)=\left[\phi_{1}(t), \phi_{2}(t), \cdots, \phi_{q}(t)\right]^{T}$ where $\left\{\phi_{1}(t), \phi_{2}(t), \cdots, \phi_{q}(t)\right\}$ are linearly independent. The inner product in $\mathcal{L}_{2}\left(t_{0}, t\right)$ is defined as $(x, y)=\int_{t_{0}}^{t} x^{T}(\tau) y(\tau) d \tau$ and the $\mathcal{L}_{2}$ norm of $x$ is given by $\|x\|_{2}=\sqrt{(x, x)}$. Then, we can show that

$$
\Delta(t)=\int_{t_{0}}^{t} \Phi(\tau) \Phi^{T}(\tau) d \tau=\left[\begin{array}{cccc}
\left(\phi_{1}, \phi_{1}\right) & \left(\phi_{1}, \phi_{2}\right) & \cdots & \left(\phi_{1}, \phi_{q}\right) \\
\left(\phi_{2}, \phi_{1}\right) & \left(\phi_{2}, \phi_{2}\right) & \cdots & \left(\phi_{2}, \phi_{q}\right) \\
\vdots & \vdots & \cdots & \vdots \\
\left(\phi_{q}, \phi_{1}\right) & \left(\phi_{q}, \phi_{2}\right) & \cdots & \left(\phi_{q}, \phi_{q}\right)
\end{array}\right]
$$

is a positive definite symmetric matrix function. ${ }^{26}$ For completeness, we present a proof. Consider a vector $\mathbf{a}(t)=\sum_{i=1}^{q} a_{i} \phi_{i}(t)$, where $\left(a_{1}, a_{2}, \cdots, a_{q}\right)$ are real scalars. Then, we obtain

$$
0 \leq\|\mathbf{a}(t)\|_{2}^{2}=\left(\sum_{i=1}^{q} a_{i} \phi_{i}(t), \sum_{j=1}^{q} a_{j} \phi_{j}(t)\right)=\sum_{i=1}^{q} \sum_{j=1}^{q} a_{i}\left(\phi_{i}, \phi_{j}\right) a_{j}=\mathbf{d}^{T} \Delta(t) \mathbf{d},
$$

where $\mathbf{d}=\left[a_{1}, a_{2}, \cdots, a_{q}\right]^{T}$. Obviously, $\Delta(t) \geq 0$. Note that $\mathbf{d}^{T} \Delta(t) \mathbf{d}=0$ if and only if 
$\left\|\sum_{i=1}^{q} a_{i} \phi_{i}(t)\right\|_{2}=0$, or equivalently,

$$
\sum_{i=1}^{q} a_{i} \phi_{i}(t)=0
$$

Since $\left\{\phi_{1}(t), \phi_{2}(t), \cdots, \phi_{q}(t)\right\}$ are linearly independent, (20) is true if and only if $\mathbf{d}=0$. Hence, we conclude that $\Delta(t)>0$. Note that this condition is known as the Persistent Excitation (PE) ${ }^{17}$.

Let $\hat{f}(t)$ be an estimate of $f(t)$ at time $t$ and given by

$$
\hat{f}(t)=\hat{\Theta}^{T}(t) \Phi(\tau), t_{0} \leq \tau \leq t
$$

where $\hat{\Theta}(t)$ is an estimate of $\Theta$ at time $t$. Then, the approximation error $\epsilon(t)$ can be defined by

$$
\epsilon(t)=\hat{\Theta}^{T}(t) \Phi(\tau)-f(\tau), t_{0} \leq \tau \leq t
$$

It should be noted that if we take the control execution period into consideration, then (21) should be modified to be

$$
\hat{f}(t)=\hat{\Theta}^{T}(t) \Phi\left(\tau-\sigma_{s}\right),
$$

where $\sigma_{s}$ is the control period. However, in this paper we assume the process delay is negligible, that is $\sigma_{s} \approx 0$. Given the approximation error at time $t$ as given in (22), we consider the following exponential cost function,

$$
J_{\epsilon}(\hat{\Theta}(t))=\frac{1}{2} \int_{t_{0}}^{t} e^{-2 \alpha(t-\tau)} \epsilon^{T}(\tau) \epsilon(\tau) d \tau
$$

where $\alpha \geq 0$ is known as the forgetting factor ${ }^{17}$ and considered as a design parameter in this study. Note that $J_{\epsilon}(\hat{\Theta})$ is a convex function of $\hat{\Theta}$ and that the exponential factor $\alpha$ is imposed to ensure the specified rate of decay for approximation error $\epsilon$. The minimum for $J_{\epsilon}(\hat{\Theta})$ can be determined from

$$
\frac{\partial J_{\epsilon}^{T}}{\partial \hat{\Theta}^{T}}=0=\int_{t_{0}}^{t} e^{-2 \alpha(t-\tau)} \Phi(\tau) \Phi^{T}(\tau) d \tau \hat{\Theta}(t)-\int_{t_{0}}^{t} e^{-2 \alpha(t-\tau)} \Phi(\tau) f^{T}(\tau) d \tau
$$

Solving for $\hat{\Theta}(t)$ at time $t$ gives

$$
\hat{\Theta}(t)=R(t) \int_{t_{0}}^{t} e^{-2 \alpha(t-\tau)} \Phi(\tau) f^{T}(\tau) d \tau
$$


where

$$
R(t)=\left[\int_{t_{0}}^{t} e^{-2 \alpha(t-\tau)} \Phi(\tau) \Phi^{T}(\tau) d \tau\right]^{-1} .
$$

It follows from (18) that $R(t)>0$. Furthermore, by utilizing the fact that $R(t) R^{-1}(t)=I$ and taking the time derivative on both sides, yields

$$
\frac{d R^{-1}(t)}{d t}=-R^{-1}(t) \dot{R}(t) R^{-1}(t)
$$

where $R^{-1}(t)$ is given by

$$
R^{-1}(t)=\int_{t_{0}}^{t} e^{-2 \alpha(t-\tau)} \Phi(\tau) \Phi^{T}(\tau) d \tau
$$

To derive the expression for $\frac{d R^{-1}(t)}{d t}$, we make use of the Leibniz's rule ${ }^{27}$ to obtain

$$
\frac{d R^{-1}(t)}{d t}=-2 \alpha R^{-1}+\Phi(t) \Phi^{T}(t)
$$

Now, we equate (29) to (27), and pre- and post-multiply by the matrix $R(t)$, we obtain

$$
\dot{R}(t)=2 \alpha R(t)-R(t) \Phi(t) \Phi^{T}(t) R(t)
$$

Similarly, taking the time derivative for (25) renders

$$
\dot{\hat{\Theta}}(t)=\dot{R}(t) \int_{t_{0}}^{t} e^{-2 \alpha(t-\tau)} \Phi(\tau) f^{T}(\tau) d \tau+R(t) \frac{d}{d t} \int_{t_{0}}^{t} e^{-2 \alpha(t-\tau)} \Phi(\tau) f^{T}(\tau) d \tau
$$

Substituting (30) for $\dot{R}(t)$ and applying the Leibniz's rule to the second term in (31), to obtain

$$
\dot{\hat{\Theta}}(t)=-R(t) \Phi(t)\left\{\Phi^{T}(t) \hat{\Theta}(t)-f^{T}(t)\right\} .
$$

Therefore, (32) and (30) together describe the estimation dynamics. ${ }^{17}$ Let the estimation error $\tilde{\Theta}(t)$ be defined by

$$
\tilde{\Theta}(t)=\hat{\Theta}(t)-\Theta, \dot{\tilde{\Theta}}(t)=\dot{\hat{\Theta}}(t),
$$

then substituting (33) into (32), and combining with (30), yields

$$
\left\{\begin{array}{l}
\dot{\tilde{\Theta}}(t)=-R(t) \Phi(t) \Phi^{T}(t) \tilde{\Theta}(t) \\
\dot{R}(t)=2 \alpha R(t)-R(t) \Phi(t) \Phi^{T}(t) R(t)
\end{array}\right.
$$


Note that Eq. (30) is a time-varying Riccati differential equation (TVRDE). By choosing

$$
V_{a}(\tilde{\Theta})=\operatorname{trace}\left\{\tilde{\Theta}^{T} R^{-1} \tilde{\Theta}\right\}>0
$$

as a candidate Lyapunov function for (34), we can show that $\tilde{\Theta}(t) \rightarrow 0$, and hence $\epsilon(t) \rightarrow 0$, as $t \rightarrow \infty$. To prove this, we take the time derivative of $V_{a}(\cdot)$ along the trajectory of $(34)$ to render

$$
\dot{V}_{a}=2 \operatorname{trace}\left\{\dot{\tilde{\Theta}}^{T} R^{-1} \tilde{\Theta}\right\}+\operatorname{trace}\left\{\tilde{\Theta}^{T} \frac{d R^{-1}(t)}{d t} \tilde{\Theta}\right\} \text {. }
$$

Now, substituting (34) and (27) into (35), and after some simple algebraic manipulations, yields

$$
\begin{aligned}
\dot{V}_{a} & =-\Phi^{T} \tilde{\Theta} \tilde{\Theta}^{T} \Phi-2 \alpha \operatorname{trace}\left\{\tilde{\Theta}^{T} R^{-1} \tilde{\Theta}\right\} \\
& =-\Phi^{T} \tilde{\Theta} \tilde{\Theta}^{T} \Phi-2 \alpha V_{a}(\tilde{\Theta}) \\
& <0
\end{aligned}
$$

for all $\tilde{\Theta} \neq 0$.

It should be noted that the forgetting factor $\alpha$ in (34) indicates a convergent rate of approximation error. Though it is treated as a design parameter, it cannot be chosen arbitrarily, since it must be chosen such that the time-varying Riccati differential equation (30) yields a positive definite symmetric solution $R(t)$ for $t>0$. For instance, large $\alpha$ may result in numerical instability when solving (30). For a given $\alpha>0$, the monotonic and orderpreserving property of $\mathrm{TVRDE}^{28}$ is utilized to ensure the existence of a positive definite symmetric solution. Let $X(t, \alpha)$ be defined by

$$
X(t, \alpha)=\int_{t_{0}}^{t} e^{-2 \alpha(t-\tau)} \Phi(\tau) \Phi^{T}(\tau) d \tau
$$

that is, $X=R^{-1}$ and $X>0$. Note that $X(t, \alpha)$ is a continuously differentiable function of $\alpha$, then by utilizing the Leibniz's rule for $\frac{\partial X}{\partial \alpha}$, we obtain

$$
\begin{aligned}
\frac{\partial X}{\partial \alpha} & =\frac{\partial}{\partial \alpha} \int_{t_{0}}^{t} e^{-2 \alpha(t-\tau)} \Phi(\tau) \Phi^{T}(\tau) d \tau \\
& =-2 \int_{t_{0}}^{t}(t-\tau) e^{-2 \alpha(t-\tau)} \Phi(\tau) \Phi^{T}(\tau) d \tau \\
& \leq 0
\end{aligned}
$$

for all $t>0$, which shows that $X(t, \alpha)>0$ is monotonically decreasing as $\alpha$ increases. Similarly, we can show that $R(t, \alpha)>0$ is an increasing function of $\alpha$. To prove this, we apply the fact that $X X^{-1}=I$ to obtain

$$
\frac{\partial X}{\partial \alpha} X^{-1}+X \frac{\partial X}{\partial \alpha}^{-1}=0
$$


hence we obtain

$$
\frac{\partial R}{\partial \alpha}=\frac{\partial X^{-1}}{\partial \alpha}=-X^{-1} \frac{\partial X}{\partial \alpha} X^{-1} \geq 0
$$

This result confirms that the fast adaption can be achieved by choosing larger $\alpha$, since $R$ is an increasing matrix function of $\alpha$.

Many known numerical schemes can be used to solve the TVRDE (30) for a given $\alpha$. For instance, one may first convert the TVRDE into coupled $q(q+1) / 2$ differential equations and use a numerical integration scheme to solve these equations for $R(t)$ at each $t$, then check if $R(t)>0$. In practice, it is important to note that the upper bound on $\alpha$ can be numerically determined by solving the TVRDE for a positive definite solution, hence the maximum achievable rate of convergence is assumed to be known when a set of basis functions $\Phi(t)$ is known.

\section{A Momentum Estimator}

We propose the following momentum estimator for (10),

$$
\dot{\hat{H}}(t)=\hat{H}(t) \times \omega(t)+u(t)+L_{c}(H-\hat{H})+\hat{f}(t),
$$

where $\hat{H}$ denotes the estimate of $H, \hat{f}$ the estimate of $f$ as defined in (21), and $L_{c}$ the estimator gain matrix to be determined. Let the momentum estimation error be defined as

$$
e(t)=\hat{H}(t)-H(t)
$$

then we can simply obtain

$$
\dot{e}(t)=\dot{\hat{H}}(t)-\dot{H}(t) .
$$

Substituting (10) and (39) into (40) yields the estimation error dynamics as

$$
\dot{e}(t)=e(t) \times \omega(t)-L_{c} e(t)+\tilde{\Theta}^{T}(t) \Phi(t),
$$

where we have applied the fact that $\hat{f}(t)-f(t)=\tilde{\Theta}^{T}(t) \Phi(t)$. Next, we show that $e(t)$ and $\tilde{\Theta}(t)$ converge to 0 at a rate of convergence $\alpha$.

Let

$$
V(e, \tilde{\Theta})=\eta e^{T} e+\underbrace{\operatorname{trace}\left\{\tilde{\Theta}^{T} R^{-1} \tilde{\Theta}\right\}}_{V_{a}}
$$

be a candidate Lyapunov function for (34) and (41), where $\eta>0$ is a parameter to be determined. Now, taking the time derivative of $V(e, \tilde{\Theta})$ along the trajectory of $(41)$ and 
(34), we obtain

$$
\dot{V}(e, \tilde{\Theta})=-2 \eta e^{T} L_{c} e+2 \eta e^{T}(e \times \omega)+2 \eta e^{T} \tilde{\Theta}^{T} \Phi+\dot{V}_{a}
$$

Since $e \times \omega$ is perpendicular to $e$, the second term above is zero. Now, substituting (36) into (42), we obtain

$$
\dot{V}(e, \tilde{\Theta})=-2 \eta e^{T} L_{c} e+2 \eta e^{T} \tilde{\Theta}^{T} \Phi-\Phi^{T} \tilde{\Theta} \tilde{\Theta}^{T} \Phi-2 \alpha V_{a}(\tilde{\Theta})
$$

Now, we can choose the observer gain $L_{c}$ to be a positive definite symmetric matrix such that $e^{T} L_{c} e \geq \lambda_{m}\|e\|^{2}$, where $\lambda_{m}$ denotes the minimum eigenvalue of $L_{c}$. More specifically, we choose $L_{c}$ such that $\lambda_{m} \geq(\alpha+\gamma)$, where $\alpha$ is the forgetting factor and $\gamma>0$ is any scalar. Then, (43) can be rewritten as

$$
\begin{aligned}
\dot{V}(e, \tilde{\Theta}) & \leq-2 \alpha \eta\|e\|^{2}-2 \alpha \operatorname{trace}\left\{\tilde{\Theta}^{T} R^{-1} \tilde{\Theta}\right\}-2 \gamma \eta\|e\|^{2}+2 \eta\|e\|\left\|\tilde{\Theta}^{T} \Phi\right\|-\left\|\tilde{\Theta}^{T} \Phi\right\|^{2} \\
& =-2 \alpha V(e, \tilde{\Theta})-2\left[\begin{array}{c}
\gamma^{\frac{1}{2}} \eta^{\frac{1}{2}}\|e\| \\
\left\|\tilde{\Theta}^{T} \Phi\right\|
\end{array}\right]^{T} \underbrace{\left[\begin{array}{cc}
1 & -\frac{1}{2} \gamma^{-\frac{1}{2}} \eta^{\frac{1}{2}} \\
-\frac{1}{2} \gamma^{-\frac{1}{2}} \eta^{\frac{1}{2}} & \frac{1}{2}
\end{array}\right]}_{M(\eta)}\left[\begin{array}{c}
\gamma^{\frac{1}{2}} \eta^{\frac{1}{2}}\|e\| \\
\left\|\tilde{\Theta}^{T} \Phi\right\|
\end{array}\right] .
\end{aligned}
$$

We note that $\dot{V} \leq-2 \alpha V$ if we can find $\eta>0$ such that $M(\eta) \geq 0$, and this is true if and only if $\eta \leq 2 \gamma$. Hence, by Definition 1, we have just demonstrated that both the momentum estimation error $e(t)$ and the disturbance estimation error $\tilde{\Theta}(t)$ are quadratically stable at a rate of convergence $\alpha$. Figure 2 shows a schematic of spacecraft attitude control block diagram with the proposed disturbance torque estimator.

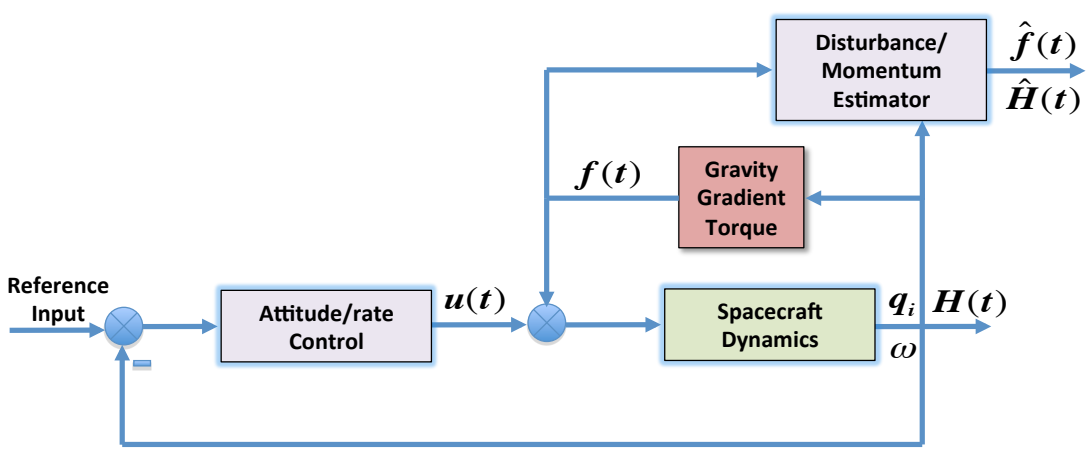

Figure 2. Schematic block diagram for disturbance torque estimation. 


\section{Numerical Simulations}

In this section, we consider a lunar orbiting small spacecraft with an orbiting rate of two hours. We assume that the spacecraft is axisymmetric and its principal moment of inertia are: $I_{x x}=110 \mathrm{~kg}-\mathrm{m}^{2}, I_{y y}=100 \mathrm{~kg}-\mathrm{m}^{2}$, and $I_{z z}=50 \mathrm{~kg}-\mathrm{m}^{2}$. It was indicated in Swei et $a l .{ }^{21}$ that the lunar gravity gradient torque is the dominant disturbance torque exerted to the spacecraft, and it is described, with respect to the body-fixed frame, as $^{23}$

$$
\left\{\begin{array}{l}
f_{x}=-6 n^{2}\left(I_{y y}-I_{z z}\right)\left(q_{1} q_{4}+q_{2} q_{3}\right)\left(1-2 q_{1}^{2}-2 q_{2}^{2}\right) \\
f_{y}=-6 n^{2}\left(I_{z z}-I_{x x}\right)\left(q_{1} q_{3}-q_{2} q_{4}\right)\left(1-2 q_{1}^{2}-2 q_{2}^{2}\right) \\
f_{z}=-12 n^{2}\left(I_{x x}-I_{y y}\right)\left(q_{1} q_{3}-q_{2} q_{4}\right)\left(q_{1} q_{4}+q_{2} q_{3}\right)
\end{array}\right.
$$

where $n$ denotes the orbiting rate and $q_{i}$ the measured attitude quaternions. Figure 3 shows the spacecraft momentum status in the inertial frame over the course of two days, where the spacecraft is slowly rotating about the body-fixed Z-axis, which is held normal to the sun vector. We can clearly see the large momentum build-up in the inertial Y-axis, while the momentum variations in the (X,Z)-axis are cyclic and much less pronounced. Therefore, when the estimated disturbance torques and the spacecraft attitude are made available, we can command an attitude maneuver for the spacecraft to align the axis of the reaction wheels momentum build-up to be opposite to the inertial Y-axis for momentum unloading.

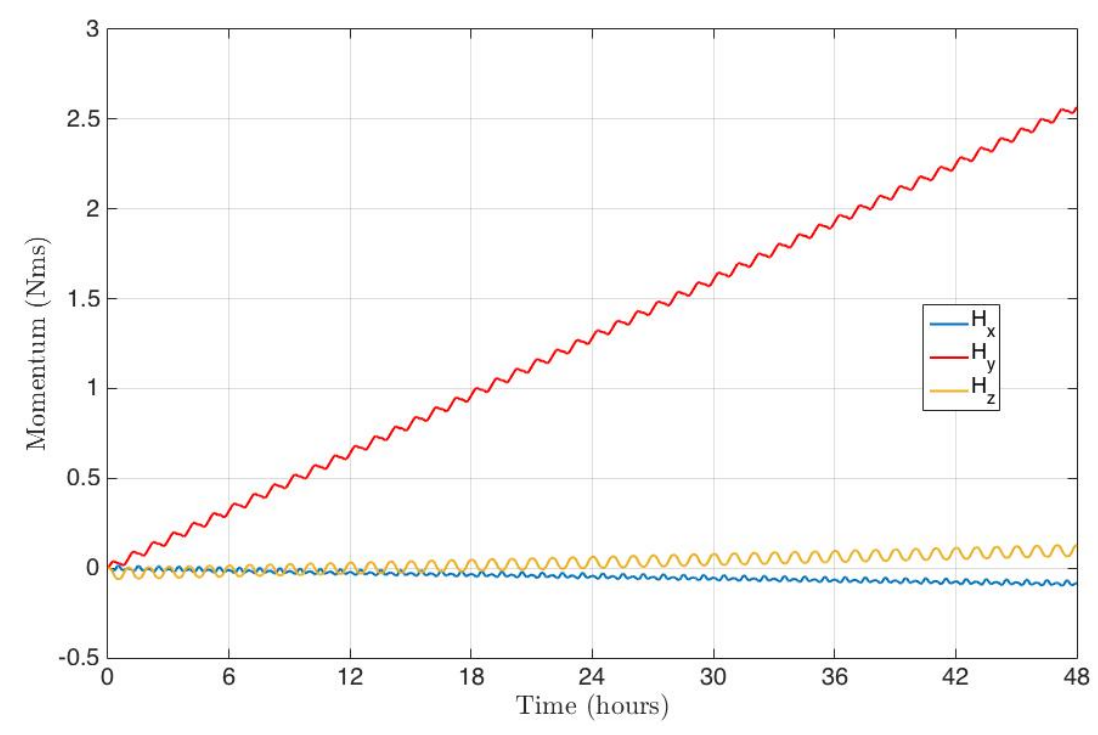

Figure 3. Spacecraft angular momentum build-up in the inertial frame.

The second order Chebyshev polynomials of the first kind are used as basis functions, that is $\Phi(t)=\left\{1, t, 2 t^{2}-1\right\}$. Two levels of forgetting factor are considered in the estimation of disturbance torques; $\alpha=0.1$ and $\alpha=0.01$. The estimator gain $L_{c}$ is chosen to be 0.25 ; 
larger $L_{c}$ was also tried but it resulted in an excessive "chattering" response. In this study, we neglected the sensor noise in the measurements, however it should be noted that the estimator gain $L_{c}$ might be further restricted if we include the measurement noise, which becomes especially relevant in the framework of Kalman filter design. Figures 4 and 5 show the adaptive estimation of disturbance torques and angular momentum when $\alpha=0.1$. As shown the proposed adaptive disturbance estimator is able to accurately predict the disturbance torques in less than two minutes. When $\alpha=0.01$, Figs. 6 and 7 show much slower rate of convergence, but still able to accurately predict the disturbance torques in about ten minutes. In addition, Figs. 8 and 9 show the time history of the nine elements in the estimation error matrix $\tilde{\Theta}(t)$, they all converge to zero at a convergent rate of $\alpha=0.1$ and $\alpha=0.01$, respectively. It should be noted that, when $\alpha=0.5$, the adaptive solutions in (34) failed to exist due to numerical instability.

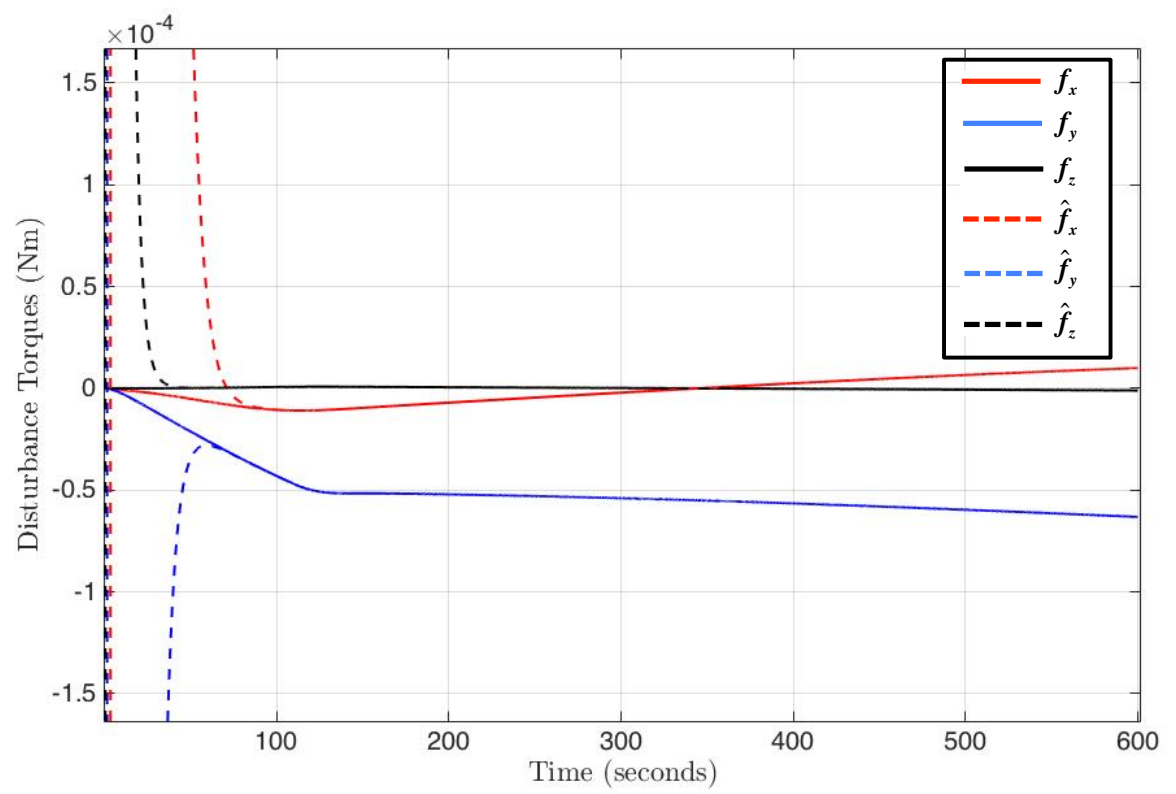

Figure 4. Actual disturbance torques and estimated disturbance torques; $\alpha=0.1$.

Next, we consider that the four reaction wheels are placed in a four-sided pyramid configuration with base angle $\beta=45 \mathrm{deg}$ and the initial rotational rates along their spin axis given by $\left[\begin{array}{llll}100 & 500 & 100 & -700\end{array}\right] \mathrm{rad} / \mathrm{s}$. The reaction wheel axial moment of inertia is assumed to be $0.001 \mathrm{~kg}-\mathrm{m}^{2}$, hence the total reaction wheel angular momentum vector equals $\left[\begin{array}{lll}0 & 0.85 & 0\end{array}\right]$ Nms. This is to simulate a lunar orbiting scenario where the total reaction wheel momentum has reached a threshold that requires de-saturation. In what follows, we will show that we can utilize the estimated environmental disturbance torque to unload the reaction wheel momentum. For easy illustration, we set the body-fixed $Y_{B}$-axis to coincide 


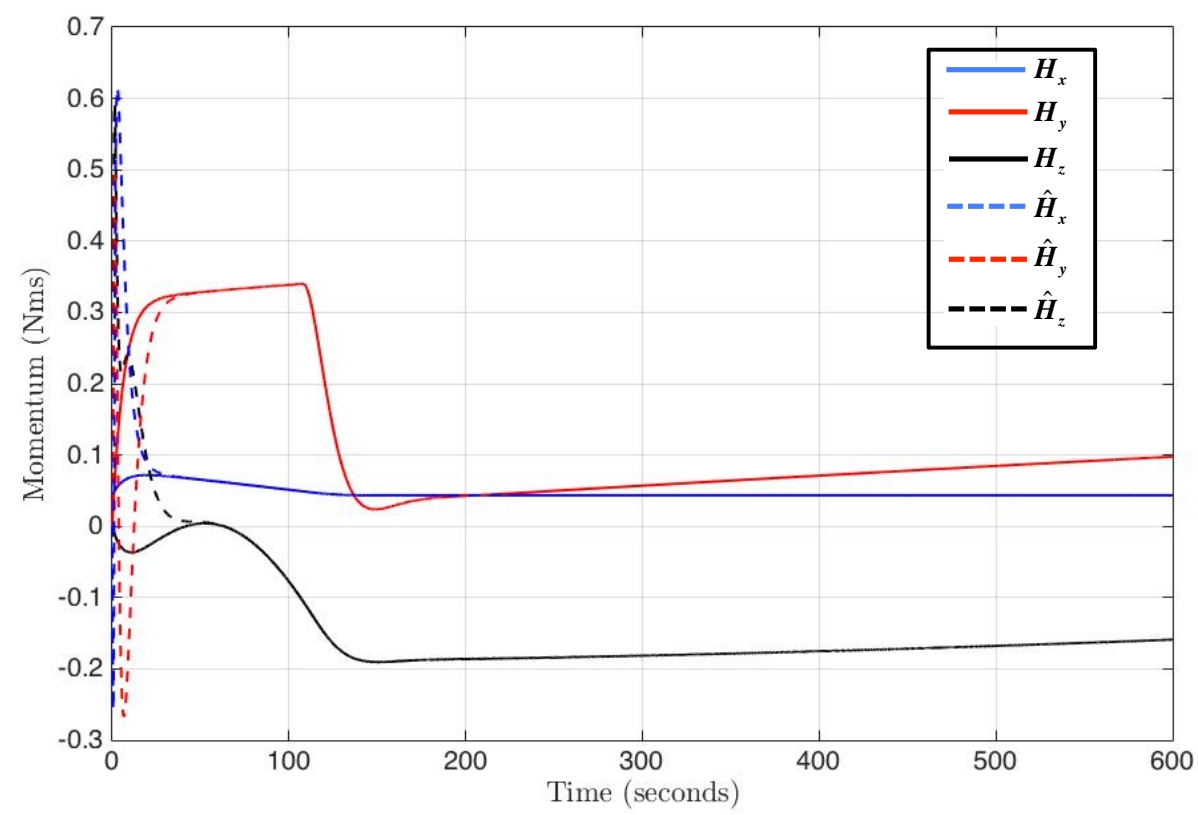

Figure 5. Actual angular momentum and estimated angular momentum; $\alpha=0.1$.

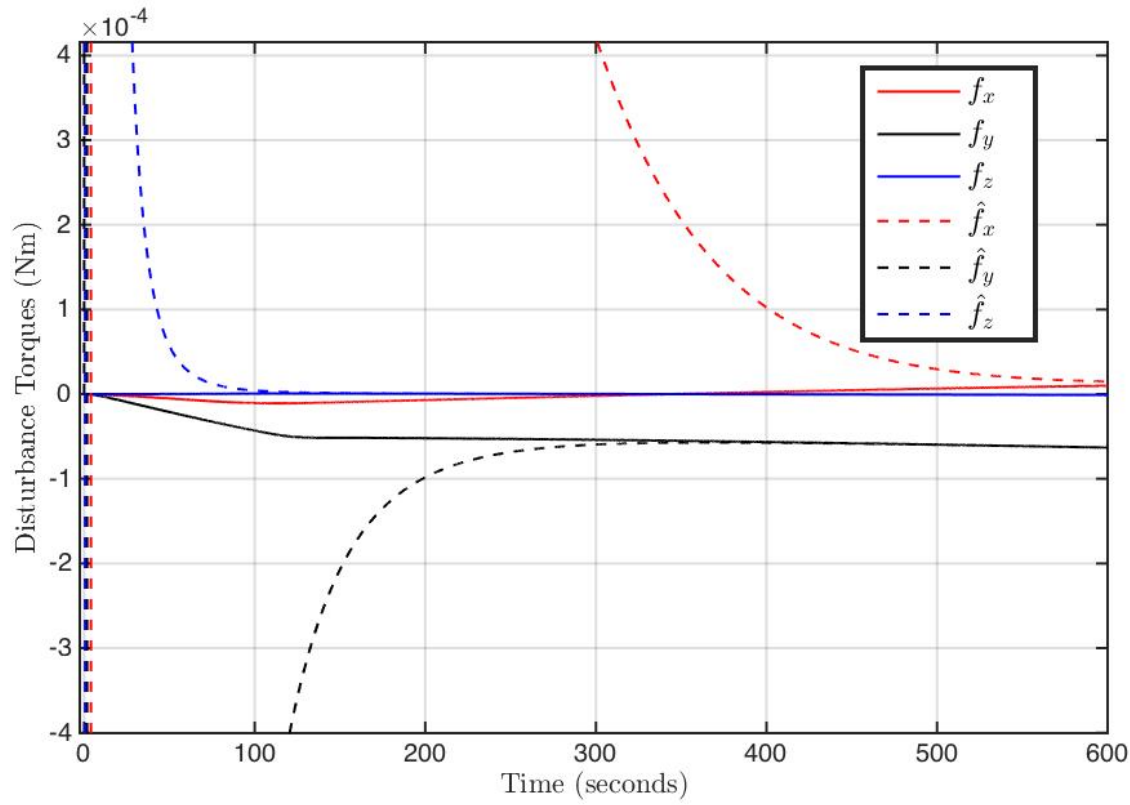

Figure 6. Actual disturbance torques and estimated disturbance torques; $\alpha=0.01$. 


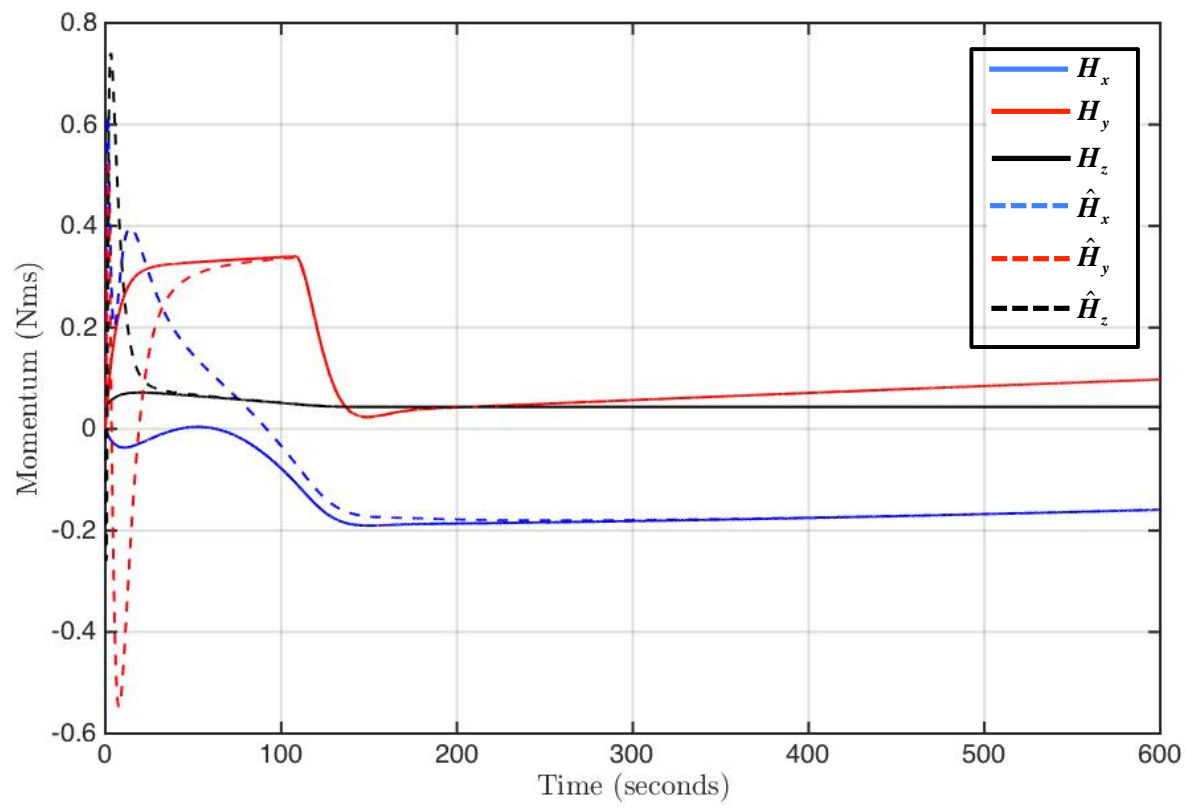

Figure 7. Actual angular momentum and estimated angular momentum; $\alpha=0.01$.

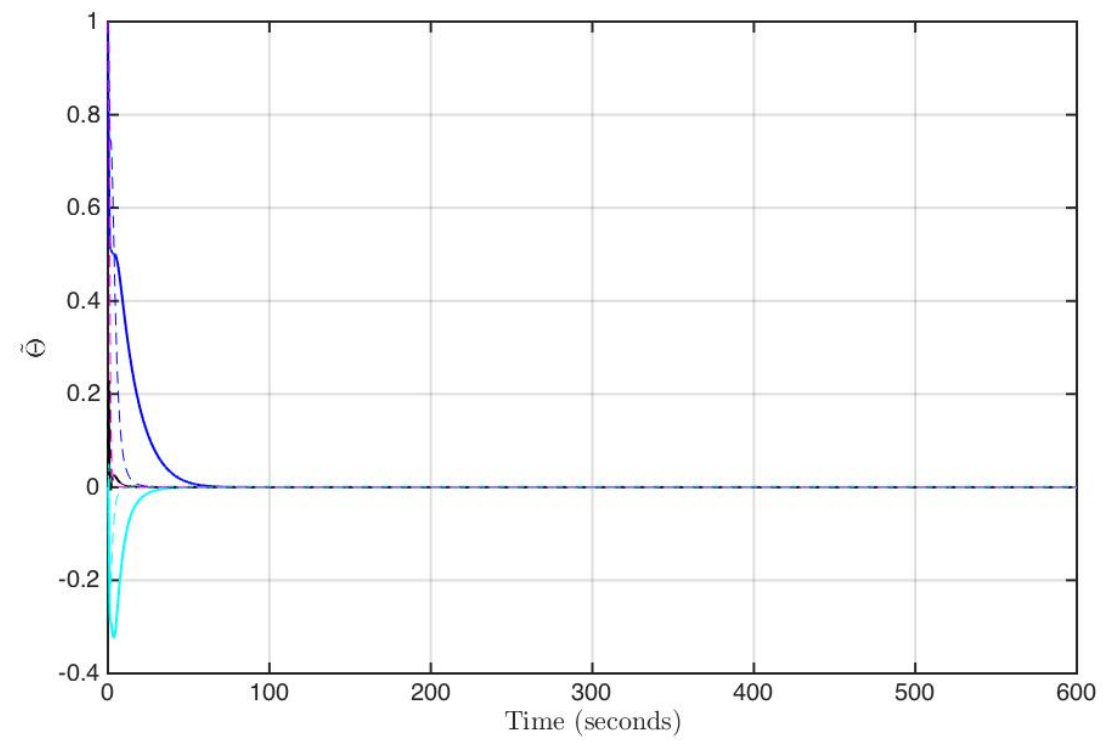

Figure 8. Elements of $\tilde{\Theta} ; \alpha=0.1$. 


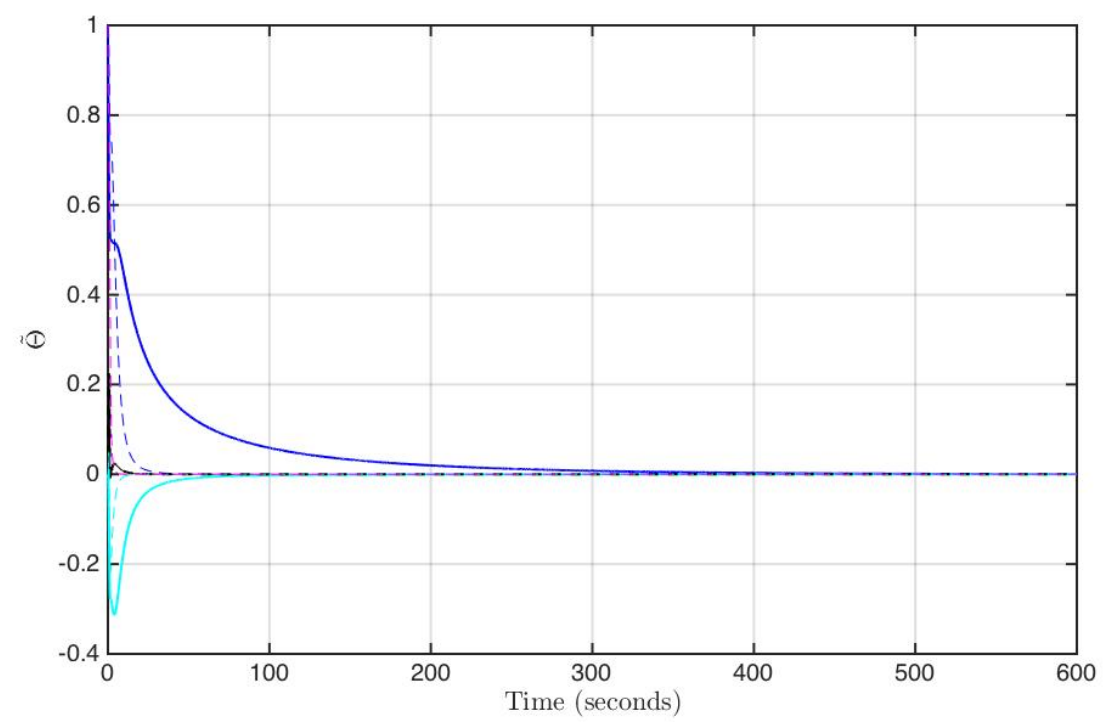

Figure 9. Elements of $\tilde{\Theta} ; \alpha=0.01$.

with the inertial $Y$-axis, hence we can execute reaction wheel momentum unloading while holding the attitude. These results are shown in Figs. 10 and 11. Figure 10 shows the gradually winding down of the reaction wheel speeds of wheel \#2 and wheel \#4, whereas Fig. 11 shows the level of reaction wheel angular momentum reduction during the first six hours or 3 orbits.

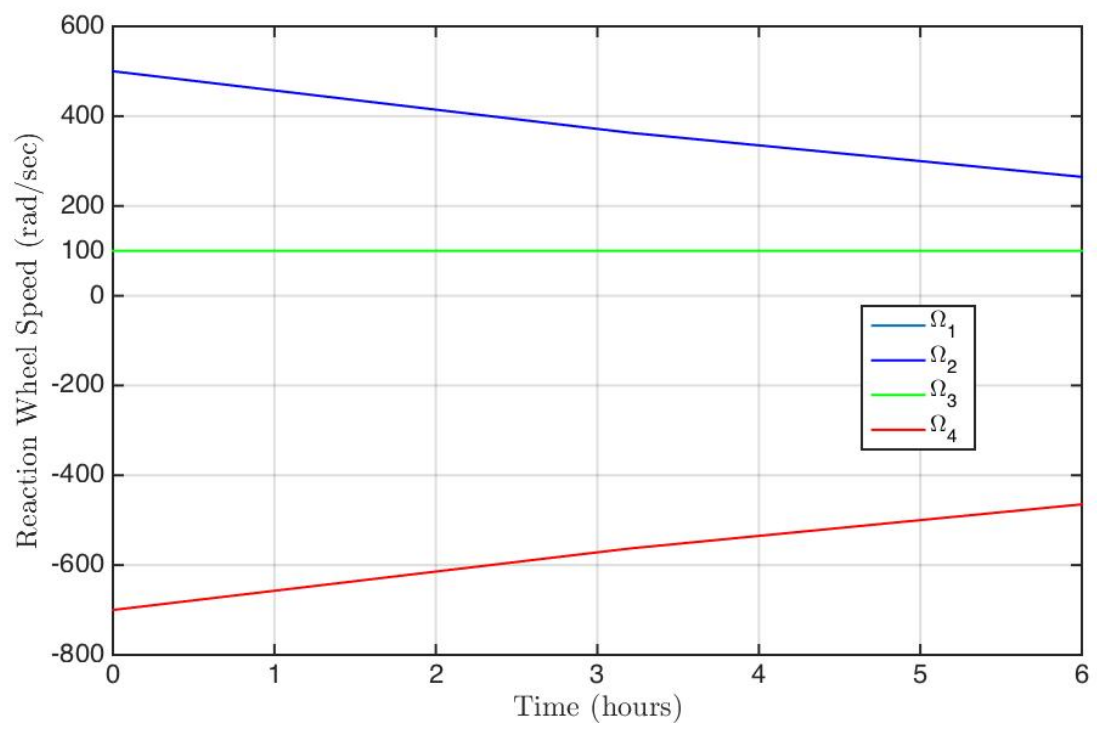

Figure 10. Reaction wheel speeds reduction during momentum unloading using disturbance torque. 




Figure 11. Reaction wheels momentum unloading using disturbance torque.

\section{Conclusions}

In this paper, a novel disturbance torque estimator was developed by utilizing the adaptive least-squares approach with a set of Chebyshev polynomials as basis functions. Given the forgetting factor $\alpha$ from the covariance updating law, an integrated adaptive scheme was proposed that guarantees the prescribed rate of convergence $\alpha$ for both spacecraft momentum and disturbance input estimation. The proposed concept was successfully demonstrated through simulations for a lunar orbiting spacecraft. The results showed that the proposed disturbance input estimator was able to predict the external disturbance torques at various prescribed convergent rate. Furthermore, the estimated disturbance torque was used for assisting spacecraft momentum management by desaturating the reaction wheel momentum.

\section{Acknowledgments}

The authors would like to thank Jesse Fusco of NASA Ames Research Center for his kind support and helpful discussions on simulation results presented in this paper. The authors would also like to thank the reviewers for their constructive comments and suggestions.

\section{References}

\footnotetext{
${ }^{1}$ http://www.nasa.gov/mission_pages/ladee/main/\#.VYDFrGSG3vN

${ }^{2}$ http://www.nasa.gov/mission_pages/cubesats/index.html

${ }^{3}$ Bhattacharyya, S.P., "Observer Design for Linear Systems with Unknown Inputs," IEEE Trans. on
} 
Auto. Cont., vol. 23, no. 3, 1978, pp. 483-484.

${ }^{4}$ Kudva, P., Viswanadham, N., and Ramakrishna A., "Observers for Linear Systems with Unknown Inputs," IEEE Trans. on Auto. Cont., vol. 25, no. 1, 1980, pp. 113-115.

${ }^{5}$ Yang, F., Wilde, R.W., "Observers for Linear Systems with Unknown Inputs," IEEE Trans. on Auto. Cont., vol. 33, no. 7, 1988, pp. 677-681.

${ }^{6}$ Guan, Y. and Saif, M., "A Novel Approach to the Design of Unknown Input Observers," IEEE Trans. on Auto. Cont., vol. 36, no. 5, 1991, pp. 632-635.

${ }^{7}$ Messaoud, R.B., Zanzouri N., and Ksouri Mekki, "Local Feedback Unknown Input Observer for Nonlinear Systems," Int. J. Innov. Comp., Inf. and Cont., vol. 8, no. 2, 2012, pp. 1145-1154.

${ }^{8}$ Park, Y., Stein, J.L., "Closed-loop State and Input Observer for Systems with Unknown Inputs," International Journal of Control, vol. 48, 1988, pp. 1121-1136.

${ }^{9} \mathrm{Tu}$, J.F., Stein, J.L., "On-Line Preload Monitoring for Anti-Friction Spindle Bearings of High-Speed Machine Tools," American Control Conference, Chicago, IL, 1992, pp. 361-369.

${ }^{10}$ Saif, M., Guan, Y., "A New Approach to Robust Fault Detection and Identification," IEEE Transactions on Aerospace and Electronic Systems, vol. 29, no. 3, 1993, pp. 685-695.

${ }^{11}$ Yang, H., Saif, M., "State Observation, Fault Detection and Isolation in Bilinear Systems," International Journal of Control, vol. 67, 1997, pp. 901-920.

${ }^{12}$ Corless, M., Tu, J., "State and Input Estimation for a Class of Uncertain Systems," Automatica, vol. 34, 1998, pp. 757-764.

${ }^{13}$ Ha, Q.P., Trinh, H., "State and Input Simultaneous Estimation for a Class of Nonlinear Systems," Automatica, vol. 40, 2004, pp. 1779-1785.

${ }^{14}$ Xiong, Y., Saif, M., "Unknown Disturbance Inputs Estimation Based on a State Functional Observer Design," Automatica, vol. 39, 2003, pp. 1389-1398.

${ }^{15}$ Liu, C.S., Peng, H., "Inverse-Dynamics Based State and Disturbance Observers for Linear TimeInvariant Systems," ASME J. of Dyna. Sys., Meas., and Control, vol. 124, 2002, pp. 375-381.

${ }^{16}$ Nguyen, N.T., "Optimal Control Modification Adaptive Law with Covariance Adaptive Gain Adjustment and Normalization," Systems $\&$ Control Letters, 61, 2012, pp. 485-494.

${ }^{17}$ Ioannou, P., Fidan, B., Adaptive Control Tutorial, SIAM, 2006.

${ }^{18} \mathrm{http}: / /$ www.nasa.gov/mission_pages/kepler/overview/index.html

${ }^{19}$ https://www.nasa.gov/mission_pages/LRO/main/index.html

${ }^{20} \mathrm{http}$ ///science.nasa.gov/missions/sdo/

${ }^{21}$ Swei, S.M., Fusco, J.C., and Nakamura, R., "Design of Sun-Safe Controllers for Lunar Atmosphere and Dust Environment Explorer," AIAA Journal of Guidance, Control, and Dynamics, vol. 39, no. 9, 2016, pp. 2022-2033, http://arc.aiaa.org/doi/abs/10.2514/1.G000270

${ }^{22}$ Sidi, M.J., Spacecraft Dynamics and Control: An Practical Engineering Approach, Cambridge University Press, 1997.

${ }^{23}$ Wie, B., Space Vehicle Dynamics and Control, AIAA Education Series, 1998.

${ }^{24}$ Corless, M.J., "Guaranteed Rate of Exponential Convergence for Uncertain Dynamical Systems," Journal of Optimization Theory and Applications, vol. 64, 1990, pp. 481-494.

${ }^{25}$ Swei, S.M., Corless, M.J., "On the Necessity of Matching Condition in Robust Stabilization," Conference on Decision and Control, Brighton, U.K., 1991. 
${ }^{26}$ Corless, M.J., Frazho, A.E., Linear Systems and Control: An Operator Perspective, Pure and Applied Mathematics, Marcel Dekker, Inc., 2003.

${ }^{27}$ Anton, H., Calculus with Analytic Geometry, 2nd edition, Wiley, 1984.

${ }^{28}$ Stokes, A.N., "A Special Property of the Matrix Riccati Equation," Bull. Austr. Math. Soc., 10, 1974, pp. $245-253$. 